\title{
IMPORTANCIA DE LA BIODIVERSIDAD LOCAL Y CULTIVADA EN LA COMPLEMENTARIEDAD ESTACIONAL DE LA DIETA DE LOS CAMPESINOS DE CALAKMUL, CAMPECHE, MÉXICO, DESPUÉS DE 20 AÑ OS DE COLONIZACIÓN
}

\author{
IMPORTANCE OF LOCAL AND CULTIVATED BIODIVERSITY IN THE \\ SEASONAL COMPLEMENTARITY OF DIET IN PEASANTS FROM CALAKMUL \\ CAMPECHE, MEXICO, AFTER 20 YEARS OF COLONIZATION
}

\author{
Francisco D. Gurri ${ }^{1}$, Mirna Vallejo-Nieto ${ }^{1}$ y Dolores O. Molina-Rosales ${ }^{1}$
}

\begin{abstract}
Se comparó la dieta y su estacionalidad en una muestra campesina de Calakmul, Campeche, México, que había mostrado cambios estacionales en su composición corporal. Se realizó un estudio quincenal de pesado y consumo de alimentos durante el ciclo agrícola de 2001-2002, que se complementó con un listado de alimentos cazados, cosechados o recolectados localmente obtenido en 2018 de informantes claves. Las kilocalorías (Kcal) ingeridas de origen local fueron significativamente mayores a las de alimentos comprados tanto en la temporada de abundancia como en la de escasez, lo que sugiere que en esta población el sistema de producción de alimentos sigue siendo más importante para la dieta que los ingresos monetarios. A pesar de las pérdidas de peso durante la temporada de escasez reportadas con anterioridad, no se encontraron diferencias en Kcal consumidas entre temporadas. Los alimentos del solar, la milpa y el monte ofrecen una dieta más diversa durante la temporada de abundancia, por lo que se concluye que los cambios observados en el fenotipo no son una consecuencia de la falta de alimentos, sino del menoscabo estacional en la complementariedad de la dieta que la comida comprada no puede subsanar.
\end{abstract}

Palabras claves: nutrición, agrodiversidad, econutrición, caza y recolección, milpa.

To study the diet of a sample of peasants from Calakmul, Campeche, Mexico who suffered seasonal changes in body composition, we conducted an observational study of dietary patterns over 24-hour periods made at 15-day intervals during the 2001-2002 agricultural cycle. The study was complemented with a list of locally hunted, harvested or gathered foods, obtained from key informant interviews in 2018. Significantly more Kilocalories (Kcal) were obtained from the local environment than from store-purchased foods in both seasons, emphasizing the dietary importance of local subsistence production over commercial endeavors. There were no Kcal differences between seasons. Food obtained from agricultural plots, backyards and woodlands offered a balanced diet, but diversity was greater during the abundance season. The results suggest that the reported weight loss during the scarcity season is not due to a lack of food, but to the deterioration of the diet's complementarity which store foods are unable to compensate for.

Key words: Nutrition, agrodiversity, eco-nutrition, hunting and gathering, milpa.

El municipio de Calakmul, en el extremo sureste del estado de Campeche en la frontera con Guatemala, nos ofrece la oportunidad de observar cómo generamos soluciones a problemas presentados por ambientes que nos son desconocidos. A diferencia de otros lugares que reciben migrantes, el sur de Calakmul se pobló a fines de los setenta cuando estaba deshabitado. En respuesta a la última reforma agraria, campesinos, peones y trabajadores urbanos de todo México viajaron a la selva del sureste, sin guías, para vivir en un ambiente que desconocían (Gurri 2010).

Parte importante de este proceso incluyó la adquisición y preparación de recursos locales ajenos a su alimentación, y la adaptación de plantas y animales que utilizaban en su lugar de origen. Este aprovechamiento de la biodiversidad nativa, así como la incorporación exitosa de los alimentos importados, puede considerarse como una aventura creativa de

1 Departamento de Ciencias de la Sustentabilidad, Laboratorio de Antropología Ambiental y Género, El Colegio de la Frontera Sur, Unidad Campeche, México. fgurri@ecosur.mx; mvallejo@ecosur.mx; dmolina@ecosur.mx 
cada unidad doméstica. Cuando hicimos el trabajo de campo que eventualmente condujo a la elaboración de este manuscrito, se estaban casando las primeras niñas que crecieron en la selva de Calakmul. Ellas llevaban consigo el conocimiento obtenido en el hogar materno para aplicarlo en los fogones de sus suegras, quienes seguramente agregaron el suyo para enriquecer sus cocinas. Con el tiempo, estos intercambios derivarán en una tradición culinaria local basada en los alimentos que las familias producen, cazan o colectan localmente.

Cada vez más, sin embargo, los alimentos locales están siendo sustituidos por dietas globalizadas que contienen productos procesados industrialmente (Cantor et al. 2018; Guthman 2011; Kraft et al. 2018). En zonas rurales estos productos se venden en tiendas de abarrotes (tienditas). Son alimentos ricos en azúcares y grasas, de bajo costo, fácil preparación y con una alta palatabilidad (Baker y Friel 2016; Biing-Hwan et al. 2014; Monteiro et al. 2011). Además, su difusión ha acarreado el despliegue de una mercadotecnia diseñada para asociarlos a necesidades sociales y afectivas que promueven su consumo y desincentivan el de los alimentos locales (Barthes 2006; EntrenaDurán y Jiménez-Díaz 2013; Mc Phail Fanger 2012).

En Calakmul, la producción agrícola para el mercado está siendo promovida por el gobierno a expensas de la producción de alimentos. Esta combinación, producción para el mercado y aumento en la oferta de alimentos industrializados suele contribuir a modificar la relación agroecológica entre el campesino y sus espacios, para socavar aún más las tradiciones culinarias locales con consecuencias negativas para la seguridad alimentaria (Chappell et al. 2013; Otero et al. 2017), la salud (Baer 1987; Fleuret y Fleuret 1980; Gurri 2011; Guthman 2011; Kennedy et al. 1992) y el ambiente (Jacques y Jacques 2012; Sohn et al. 1999). Esta combinación puede ser aún más peligrosa en Calakmul, donde una tradición culinaria basada en los recursos cazados, recolectados o cosechados en su entorno no ha tenido tiempo de despegar.

En este trabajo, documentamos la importancia de los alimentos que los habitantes de Calakmul cazan, recolectan, cosechan o crían. Notamos la distribución de plantas y animales nativos e importados en los diferentes espacios de su nuevo sistema agroecológico para entender cómo se apropiaron de su nuevo ambiente. Finalmente, comparamos la contribución calórica de los alimentos obtenidos en estos espacios con la de los productos comprados, para ver si después de 20 años de colonización los campesinos de Calakmul generaron una dieta basada en el uso diverso de su agroecosistema o prefirieron comprar sus alimentos en las tiendas, con ingresos generados por la venta de productos agrícolas.

\section{Área de Estudio}

La investigación se llevó a cabo en siete comunidades del municipio de Calakmul, Campeche, situado al sur de la región de la Península de Yucatán, entre los paralelos $19^{\circ} 12^{\prime} 00^{\prime \prime}$ y $17^{\circ} 48^{\prime} 39^{\prime \prime}$ de latitud norte y los meridianos $89^{\circ} 09^{\prime} 04^{\prime \prime}$ longitud este y $90^{\circ}$ 29' 05" longitud oeste de Greenwich (Figura 1). El terreno es de tipo cárstico con suelos someros, lo que le confiere una alta infiltración. La vegetación predominante es selva alta y mediana perennifolia. El clima es tropical subhúmedo con lluvias en verano que van de junio a noviembre (Martínez y Galindo 2002). Las cosechas se dan a finales de octubre y principios de noviembre y la mayoría de las frutas se cosechan durante las secas, en los primeros meses del año, por lo que los campesinos tienen que adaptarse a una larga temporada de escasez que va de mayo a octubre (Alayón-Gamboa y Gurri 2007).

En 1970, el gobierno federal promovió la formación de nuevos ejidos ${ }^{1}$ e inauguró la carretera Escárcega-Chetumal conectando el sur de la Península con el resto del país. Campesinos provenientes de 23 estados, pero particularmente de Guanajuato, Durango, Coahuila, Veracruz, Tabasco y Chiapas, respondieron y colonizaron el área (Gurri 2007). En la actualidad, esta parte de la selva del sur de Campeche alberga a una población que creció de 4.000 personas en 1970 a 26.882 en 2010 (Instituto Nacional de Estadística y Geografia [INEGI] 2011).

A pesar del rápido crecimiento poblacional, la densidad de población se mantuvo baja $-1,92$ personas por $\mathrm{km}^{2}$ - (Secretaría de Desarrollo Social [SEDESOL] 2013). Las familias estudiadas cultivan su propia tierra, ya sea en terrenos privados, ejidales o prestados. La agricultura de temporada se realiza en dos parcelas que juntas abarcan 2,85 ha. Estas están bien drenadas en tierra plana u ondulada con una profundidad de 5 a $50 \mathrm{~cm}$. La roza-tumba-quema es la estrategia preferida de cultivo. La selva virgen suele ser tumbada para el cultivo de chile jalapeño (Capsicum annum). Después de la primera cosecha de chile, la parcela se utiliza para sembrar milpa ${ }^{2}$ uno o dos años. Cualquier parcela recién abierta 


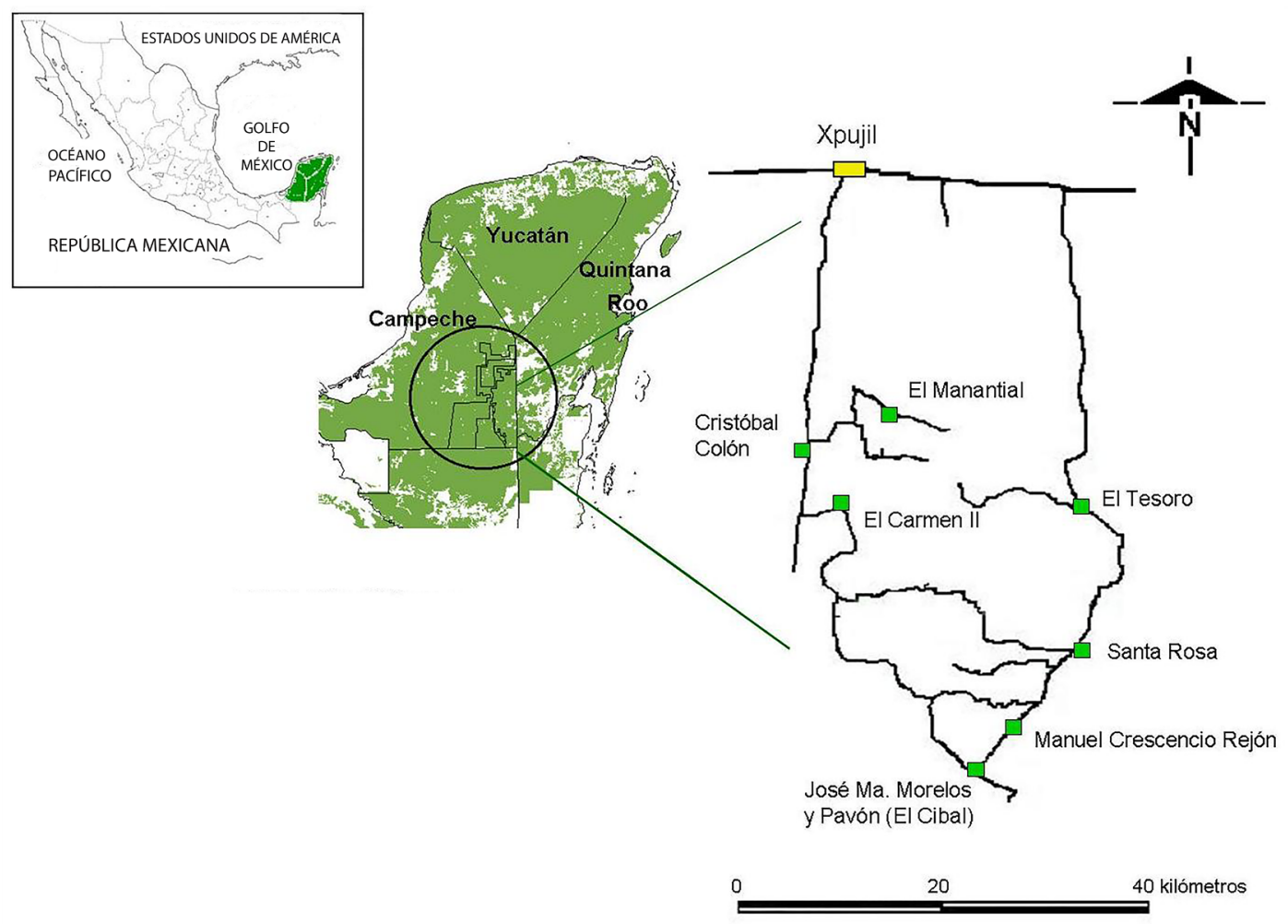

Figura 1. Mapa de localización de la región de estudio. Se indican las comunidades donde se obtuvieron los datos. Tomado de Gurri et al. 2002.

Map of the study area showing the towns where the data was collected. Taken from Gurri et al. 2002.

al cultivo será usada por un máximo de tres ciclos agrícolas y dejada descansar por un mínimo de cinco años y, usualmente, por más de 10. La mayoría de los productos de la milpa son cosechados para el consumo en el hogar y para obtener los subsidios del gobierno (PROCAMPO). Relativamente pocos insumos se invierten en la milpa, y de sus productos, solamente la semilla de la calabaza (chihua) se comercializa. La producción de chile jalapeño, por el contrario, se destina completamente al mercado y su cultivo requiere de buena tierra, altos insumos en pesticidas y herbicidas, así como atención a lo largo de la temporada de crecimiento. Una vez que ha sido cosechado y empacado, el producto es comprado por intermediarios o coyotes, quienes lo venden fuera del área (Gurri et al. 2001).

\section{Material y Métodos}

Durante el ciclo agrícola 2001-2002 el equipo del actual laboratorio de Antropología Ambiental y
Género visitó 16 unidades domésticas. Cada unidad doméstica fue visitada en tres meses distintos. Esto nos permitió tener cuatro registros de consumo y pesado de alimentos durante cada mes del año. En cada visita se registró y pesó todo lo que consumieron los miembros de la unidad doméstica por un periodo de 24 horas, usando la técnica de pesados y medidas de Lieberman (1986) y se preguntó el origen de todos los alimentos registrados.

Una lista de todo lo consumido se utilizó para diseñar un cuestionario de frecuencia de alimentos. Este se aplicó a amas de casa y otros personajes claves en las comunidades para confirmar el origen de los comestibles, así como las fechas en los que se comían. Con los datos generados por ambos métodos se elaboró un "diccionario de alimentos" (Gurri et al. 2004). La composición de los víveres se obtuvo de las tablas del Instituto Nacional de Ciencias Médicas y Nutrición Salvador Zubirán [INCMNSZ] (1998) y de análisis bromatológicos realizados en el laboratorio de alimentos de El Colegio de la Frontera Sur. 
Las especies que se cosecharon, cazaron o recolectaron en Calakmul fueron clasificadas como alimentos de origen animal, vegetal u hongos. Los últimos fueron incluidos después como vegetales siguiendo la Norma Oficial Mexicana NOM-043SSA2-2012 (Secretaría de Salud [SSA] 2013). Las plantas y animales de la península de Yucatán, así como aquellos que forman parte del repertorio culinario prehispánico mesoamericano, fueron incluidas como nativas, y las demás como introducidas. Una prueba de $X^{2}$ se utilizó para determinar si diferencias en los espacios de obtención de animales y vegetales consumidos eran significativamente distintas, y otra sirvió para determinar si los espacios albergaban proporciones diferentes de especies nativas o importadas por los migrantes.

Se calcularon las kilocalorías (Kcal) de cada platillo consumido durante la visita y se sumaron para obtener el total de Kcal consumidas por individuo por día. Estos consumos se promediaron para la temporada de abundancia y la de escasez. Excepto por los alimentos consumidos crudos, la mayoría forma parte de platillos que integran ingredientes comprados y obtenidos en el ambiente. Para estimar la importancia calórica de cada grupo sin tener que clasificar todos los ingredientes pesados, se seleccionó un alimento al azar de cada platillo. Los ingredientes escogidos, por lo tanto, pueden considerarse una muestra representativa de los ingredientes consumidos. Cada ingrediente seleccionado fue clasificado como obtenido en el ambiente o comprado. Las Kcal ingeridas por un individuo durante una visita se sumaron por categoría para obtener el número de Kcal provenientes del ambiente o compradas y consumidas por individuo por día. Estas fueron promediadas por temporada agrícola. Pruebas Wilcoxon de rangos con signo de muestras relacionadas fueron utilizadas para comparar, primero las Kcal totales por estación agrícola, y luego el promedio de Kcal obtenidas del ambiente con las compradas en cada temporada. En este análisis solamente se incluyeron individuos cuyos consumos fueron registrados en ambas temporadas.

\section{Resultados}

Durante nuestro periodo de observación, las familias de Calakmul consumieron 174 alimentos. De estos casi el 59\%, 102, pertenecen a 83 especies que se obtuvieron del ambiente local. Los habitantes de Calakmul los cazaron en el bosque, la milpa y el chilar con ayuda de perros de caza, rifles y pistolas calibre 22. Otros los pescaron en los cuerpos de agua con anzuelos, redes, y en el caso de las tortugas; con las manos. Finalmente, aunque en algunos chilares se usaron tractores, la mayoría de los alimentos de origen vegetal en la milpa y el solar se sembraron y cosecharon con instrumentos de labranza tradicional como el espeque, la coa, el azadón, el machete, las manos; y en los solares se alcanzaron las frutas con bajador (Figura 2).

La Tabla 1 muestra el número de especies utilizadas por los habitantes de Calakmul en su alimentación. Están divididas por origen, reino y el espacio donde fueron recolectadas, cosechadas, criadas, pescadas o cazadas. Se incluyó y clasificó la leche materna (Homo sapiens) como alimento animal del solar y las dos especies de hongos encontradas, Basidiomycetes sp. y Ustilago maydis (huitlacoche), se clasificaron como vegetales. La mayoría de las especies provienen del solar $(58,8 \%)$, seguido de la parcela $(23,5 \%)$ y el restante $(17,6 \%)$ se caza o colecta en el bosque. Sin embargo, la distribución por espacio es significativamente distinta entre animales y plantas $\left(X^{2}=18.084_{2 \text { d.f. }} \alpha<0.001\right)$. Más de la mitad de las variedades de plantas se colectan en el solar $(66,2 \%)$, mientras que el $58 \%$ de las especies animales que forman parte de la dieta son perseguidas en el bosque o se atrapan en la parcela cuando son atraídas por los cultivos.

Existen diferencias significativas $\left(X^{2}=28.348,2\right.$ d.f. $\alpha<0,001)$ entre el lugar de origen de las especies y el espacio de donde se obtienen. Las especies nativas se distribuyen equitativamente en los tres espacios, mientras que todas las introducidas, excepto la mojarra, son domesticadas y se encuentran exclusivamente en la milpa y el solar. Las 17 especies de animales que se cazan en la parcela y el bosque son originarias de y viven en la selva de Calakmul. Los campesinos salen a cazar a 12 de estas con perros y escopetas, y las restantes se cazan incidentalmente en la parcela o con trampas, en caso de que estén atacando el cultivo. Este suele ser el caso del tejón (Nasua narica) y del sereque (Dasyprocta punctata). Los animales introducidos representan el 35,5\% de todas las especies animales consumidas y todos se crían en el solar.

El 52\% de las plantas son nativas. Solo cinco, es decir, el 7\% de todas, se recolectan en el bosque. La gran mayoría de las plantas nativas representan a los cultivos tradicionales mesoamericanos y sus 


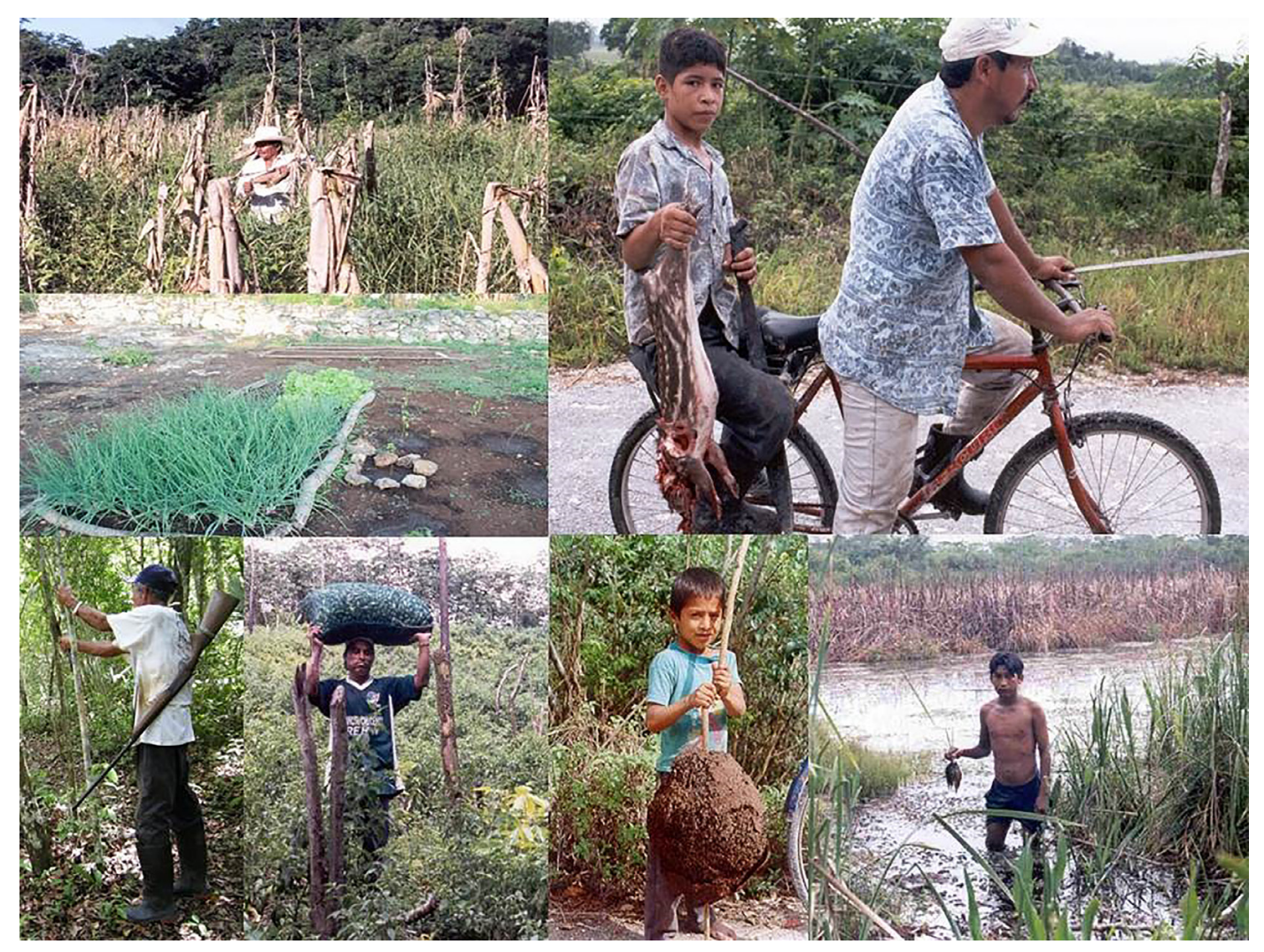

Figura 2. Nichos donde se obtienen los alimentos en Calakmul, Campeche.

Niches where foods are obtained in Calakmul, Campeche.

Tabla 1. Especies consumidas en Calakmul, Campeche, por reino, lugar de origen y espacio en el ambiente donde se obtiene. Species consumed in Calakmul, Campeche by kingdom, place of origin and space in the environment where it was obtained.

\begin{tabular}{|c|c|c|c|c|c|c|c|c|c|}
\hline \multirow[t]{3}{*}{ Reino } & \multirow[t]{3}{*}{ Origen } & \multicolumn{8}{|c|}{ Espacio donde se obtienen los alimentos } \\
\hline & & \multicolumn{2}{|c|}{ Monte } & \multicolumn{2}{|c|}{ Parcela } & \multicolumn{2}{|c|}{ Solar } & \multicolumn{2}{|c|}{ Total } \\
\hline & & $\mathrm{n}$ & $\%$ & $\mathrm{n}$ & $\%$ & $\mathrm{n}$ & $\%$ & $\mathrm{n}$ & $\%$ \\
\hline \multirow{3}{*}{ Animal } & Nativa & 12 & 38,7 & 5 & 16,1 & 2 & 6,5 & 19 & 18,63 \\
\hline & Introducida & 1 & 3,2 & 0 & 0 & 11 & 35,5 & 12 & 11,76 \\
\hline & Total & 13 & 41,9 & 5 & 16,1 & 13 & 41.9 & 31 & 30,39 \\
\hline \multirow{3}{*}{ Vegetal } & Nativa & 5 & 7 & 14 & 19,7 & 18 & 25,4 & 37 & 36,27 \\
\hline & Introducida & 0 & 0 & 5 & 7 & 29 & 40,8 & 34 & 33,33 \\
\hline & Total & 5 & 7 & 19 & 26,8 & 47 & 66,2 & 71 & 69,61 \\
\hline \multirow{3}{*}{ Total } & Nativa & 17 & 16,7 & 19 & 18,6 & 20 & 19,6 & 56 & 54,9 \\
\hline & Introducida & 1 & 1 & 5 & 4,9 & 40 & 39,2 & 46 & 45,1 \\
\hline & Total & 18 & 17,6 & 24 & 23,5 & 60 & 58,8 & 102 & \\
\hline
\end{tabular}

$X 2=18.084,2$ d.f. $\alpha<0,001$ entre el reino de las especies y el espacio donde se obtienen.

$X^{2}=28.348$,2d.f. $\alpha<0,001$ ) entre lugar de origen de las especies y el espacio donde se obtienen. 
plantas asociadas, y se siembran en las parcelas como parte de la milpa o en los solares (19,7 y $25,4 \%$ respectivamente). Un $26,3 \%$ de las plantas sembradas en las parcelas son introducidas, pero el espacio donde más se han incorporado especies foráneas es en el solar. De hecho, de las 71 plantas consumidas por los campesinos de Calakmul, 40,8\% son introducidas y se encuentran en el solar.

La Tabla 2 muestra los alimentos obtenidos por los campesinos de Calakmul, clasificados por grupo de alimento de acuerdo a la Organización de las Naciones Unidas para la Agricultura y la Alimentación [FAO] (Kennedy et al. 2013:23-24) y por estación agrícola. Todos los grupos se encuentran representados a lo largo del año. La variedad de alimentos, sin embargo, es mayor durante la temporada de abundancia que cubre la estación de secas entre ambas temporadas. La mayoría de las especies de verduras, semillas y legumbres se dan a finales de octubre, que es cuando termina la temporada de escasez. Las diferencias más importantes se producen en los cereales y frutas. La cosecha de los primeros en combinación con las verduras, semillas y legumbres de octubre marcan el inicio de la temporada de abundancia. Las frutas se recogen a lo largo de la temporada de abundancia durante la época de secas.

Tabla 2. Clasificación de alimentos locales por estación de acuerdo a la FAO (Kennedy et al. 2013).

FAO classification of local foods by season (Kennedy et al. 2013).

\begin{tabular}{|c|c|c|c|c|}
\hline \multirow{2}{*}{$\begin{array}{l}\text { Grupo de } \\
\text { alimentos }\end{array}$} & \multicolumn{4}{|c|}{ Temporada agrícola } \\
\hline & Abundancia & Escasez & Todo el año & Total \\
\hline Carnes & 2 & 3 & 21 & 26 \\
\hline Cereales & 3 & - & 2 & 5 \\
\hline $\begin{array}{l}\text { Especias y } \\
\text { condimentos }\end{array}$ & - & - & 10 & 10 \\
\hline Frutas & 12 & 3 & 11 & 26 \\
\hline $\begin{array}{l}\text { Leche y } \\
\text { productos } \\
\text { lácteos }\end{array}$ & - & - & 3 & 3 \\
\hline $\begin{array}{l}\text { Legumbres } \\
\text { nueces y } \\
\text { semillas }\end{array}$ & 2 & 4 & 1 & 7 \\
\hline Verduras & 6 & 5 & 10 & 21 \\
\hline $\begin{array}{l}\text { Pescados y } \\
\text { mariscos }\end{array}$ & - & 2 & 0 & 2 \\
\hline $\begin{array}{l}\text { Raíces y } \\
\text { tubérculos }\end{array}$ & - & 2 & 0 & 2 \\
\hline Total & 25 & 19 & 58 & 102 \\
\hline
\end{tabular}

Durante el ciclo agrícola 2001-2002, se hicieron 268 observaciones de ingesta y pesado de alimentos. Participaron 113 individuos de 16 unidades domésticas. Excepto por una que abandonó el estudio después de dos visitas, cada unidad doméstica fue visitada tres veces. La Tabla 3 muestra el número de individuos observados por visita y agrupados por temporada agrícola. Fueron tres visitas, y a pesar del abandono de una unidad doméstica durante la tercera visita, se obtuvieron 134 observaciones en cada temporada agrícola. La Tabla 4 muestra la distribución por edad y sexo de los participantes en el estudio. Se trata de familias jóvenes. El 54\% de sus miembros son menores de 30 años y más del $70 \%$ son menores de 40. El consumo calórico es diferente por edad y sexo, por lo que es usual comparar grupos similares demográficamente, por lo tanto la tabla se presenta solo como referencia para estudios que quieran contrastar sus datos con los aquí presentados. Este diseño, sin

Tabla 3. Número de individuos censados por visita y temporada. Number of individuals studied by visit and season.

\begin{tabular}{cccc}
\hline Visita & \multicolumn{4}{c}{ Temporada agrícola } \\
\hline & Abundancia & Escasez & Total \\
\hline 1 & 0 & 92 & 92 \\
2 & 51 & 42 & 93 \\
3 & 83 & 0 & 83 \\
Total & 134 & 134 & 268 \\
\hline
\end{tabular}

Tabla 4. Pirámide poblacional de la muestra durante la temporada agrícola 2001-2 en Calakmul, Campeche. Sample population pyramid during the 2001-2 agricultural season in Calakmul, Campeche.

\begin{tabular}{llll}
\hline Grupos de edad & \multicolumn{3}{c}{ Sexo } \\
\hline 0 a 4,99 & 8 & Femenino & Total \\
5 a 9,99 & 8 & 8 & 16 \\
10 a 14,99 & 10 & 3 & 11 \\
15 a 19,99 & 10 & 7 & 21 \\
20 a 24,99 & 6 & 6 & 17 \\
25 a 29,99 & 0 & 1 & 12 \\
30 a 34,99 & 3 & 7 & 1 \\
35 a 39,99 & 3 & 5 & 10 \\
40 a 44,99 & 4 & 1 & 8 \\
50 a 54,99 & 2 & 1 & 5 \\
55 a 59,99 & 1 & 2 & 3 \\
60 o mayor & 3 & 3 & 6 \\
\hline Total & 58 & 55 & 113 \\
\hline
\end{tabular}


embargo, es longitudinal. Se comparan las diferencias entre los mismos individuos por temporada, por lo que se puede usar toda la muestra. Los promedios totales presentados no representan el consumo calórico total de ningún grupo etario en particular. La Tabla 5 muestra el número de ingredientes contenidos en todos los platillos que se consumieron durante la temporada agrícola 2001-2. Más de la mitad (72,3\%)

Tabla 5. Número de ingredientes en cada platillo muestreado durante la temporada agrícola 2001-2 en Calakmul, Campeche.

Number of ingredients per platter sampled during the 2001-2 agricultural season in Calakmul, Campeche.

\begin{tabular}{|c|c|c|c|}
\hline $\begin{array}{l}\text { Número de } \\
\text { ingredientes }\end{array}$ & Frec. & $\%$ & $\%$ acum. \\
\hline 1 & 916 & 42,1 & 42,1 \\
\hline 2 & 657 & 30,2 & 72,3 \\
\hline 3 & 178 & 8,2 & 80,5 \\
\hline 4 & 150 & 6,9 & 87,4 \\
\hline 5 & 100 & 4,6 & 92,0 \\
\hline 6 & 73 & 3,4 & 95,4 \\
\hline 7 & 45 & 2,1 & 97,4 \\
\hline 8 & 20 & 0,9 & 98,3 \\
\hline 9 & 11 & 0,5 & 98,9 \\
\hline 10 & 14 & 0,6 & 99,5 \\
\hline 11 & 9 & 0,4 & 99,9 \\
\hline 12 & 2 & 0,1 & 100,0 \\
\hline Total & 2.175 & 100 & \\
\hline
\end{tabular}

$\overline{\text { Media }}=2,36,1,9$ DE.

contenía entre uno y dos elementos. Menos del 5\% contenían más de seis. El promedio de ingredientes contenido por platillo es 2,36.

La contribución a la dieta de los alimentos cosechados, cazados o recolectados en Kcal se compara en la Tabla 6 con la de los alimentos comprados. Se hicieron tres pruebas de Wilcoxon para muestras relacionadas en 88 individuos registrados en ambas temporadas. La primera prueba contrapone el total de calorías promedio consumidas durante la abundancia con las de escasez. La segunda prueba contrasta los alimentos obtenidos del ambiente con los comprados en la temporada de abundancia y la última lo hace con los de la temporada de escasez.

No hay diferencias significativas entre el promedio de Kcal consumidas por visita durante
Tabla 6. Kilocalorías consumidas por individuo por visita por estación y origen del alimento.

Kilocalories consumed by individual per visit, season and food source.

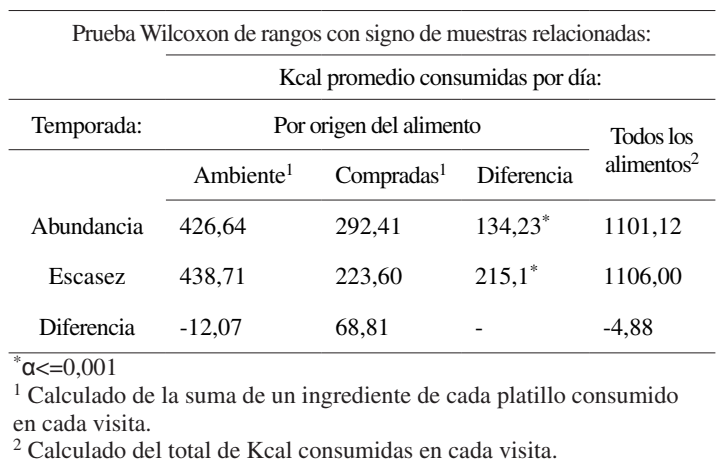

las temporadas de abundancia y de escasez. Las Kcal obtenidas de alimentos del bosque, milpa o solar, por otra parte, son significativamente mayores a las de los comprados tanto en la temporada de abundancia como en la de escasez. La diferencia en la temporada de abundancia es de $134.23 \mathrm{Kcal}$ y en la de escasez, de $215.1 \mathrm{Kcal}$.

\section{Discusión}

Diferentes autores han asociados la deslocalización de las dietas campesinas tradicionales y sus consecuencias nutricionales a la oferta de productos industrializados. Éstos incluyen una proliferación de tiendas de abarrotes con una oferta limitada (BiingHwan et al. 2014; Bustillos et al. 2009; Liese et al. 2007; Sharkey et al. 2012; Sharkey et al. 2013) y una mercadotecnia especializada diseñada para generar con el tiempo cambios en los hábitos alimentarios (Barthes 2006; Entrena-Durán y Jiménez-Díaz 2013; Leatherman y Goodman 2005; Marín Cárdenas et al. 2014; Mc Phail Fanger 2012; Pérez Izquierdo et al. 2012). Estas explicaciones, sin embargo, son pasivas. Desde el siglo pasado existen numerosos estudios de caso que sugieren que el empobrecimiento de la dieta campesina y la incorporación de los alimentos industrializados no dependen de la oferta de estos últimos, sino de la adopción de nuevos sistemas de vida y del descuido o abandono de sistemas desarrollados alrededor de un cultivo básico (Baer 1987; Cantor et al. 2018; DeWalt 1993; Dewey 1981; Fleuret y Fleuret 1980; Gurri 2011; Otero Prevost et al. 2017). Este abandono también reduce la variedad de plantas asociadas a este, así como animales domésticos o 
cazados que forman parte del sistema y que proveen a la dieta de los campesinos con fuentes adicionales de vitaminas y proteínas (Messer 1977; Oomen 1971; Terán y Rasmussen 2009; Fanzo et al. 2013; M'Kaibi et al. 2015). Esta reducción en la diversidad de la dieta se ve reflejada en la biodiversidad del sistema y afecta negativamente la seguridad alimentaria (Chappell et al. 2013).

En 20 años de colonización, los campesinos de Calakmul han aprovechado todos los espacios de su ecosistema y combinado especies introducidas con nativas. El origen de los alimentos en cada uno refleja historias de manejo distintas, que se entrelazan en una estrategia adaptativa que involucra a toda la familia y encuentra sus raíces en la América precolombina. A 500 años de la conquista, en Calakmul, la agricultura de roza-tumba-quema sigue dependiendo de las especies que se domesticaron en Mesoamérica. En el bosque se siguen cazando los animales que seguramente formaban parte de la dieta de los campesinos mayas precolombinos de la zona (Götz 2014; MariacaMéndez 2015; Varela Scherrer y Trabanino 2017). Éstos se complementan con los que son cazados en las parcelas, atraídos por los productos de la milpa. Ninguno de ellos es domesticado. Algunos son "adoptados" como mascotas y suelen ser presumidos y no sacrificados como alimento. Por lo menos no inmediatamente (Figura 3).

Mientras que los hombres han retomado el sistema tradicional en las parcelas, las mujeres en los solares han introducido plantas y animales exógenos e incorporado algunas especies nativas de la selva de Calakmul (Alayón-Gamboa y Gurri-García 2008; Neulinger et al. 2013). La caza es excitante y es celebrada por la familia. La mayoría de los platillos con carne o productos animales como el huevo, sin embargo, provienen de animales domésticos introducidos que mujeres y niños crían en el solar. En efecto, es el solar una especie de laboratorio donde mujeres, niños y ancianos fomentan la agrodiversidad que proporciona a la familia acceso a todos los grupos de alimentos necesarios para mantener una dieta balanceada (Lope-Alzina, 2017). Con el trabajo del solar, las mujeres no solo permiten que la unidad doméstica sea autosuficiente, también las empodera (Perea 2011). Como en toda América septentrional, el control de los productos del solar les pertenece. $\mathrm{Su}$ ingreso puede ser gastado en las necesidades de sus hijos y en productos para ellas, sin requerir la aprobación de sus esposos (Alayón-Gamboa y GurriGarcía 2008; Chablé-Can et al. 2007) (Figura 4).

Los agricultores que dependen de las lluvias suelen estar expuestos a temporadas de escasez. Esta se suele asociar a pérdidas de peso en toda la población y un retraso en el crecimiento (Ferro-Luzzi et al. 1990; Golden et al. 2019; Hirvonen et al. 2015; Leonard 1991). Lo mismo sucede con los campesinos de esta muestra (Alayón-Gamboa y Gurri 2007 para evaluación de estado nutrimental). A pesar de eso, no encontramos diferencias significativas en el consumo promedio de calorías entre temporadas y en ambas el consumo diario resultó estar dentro de los parámetros

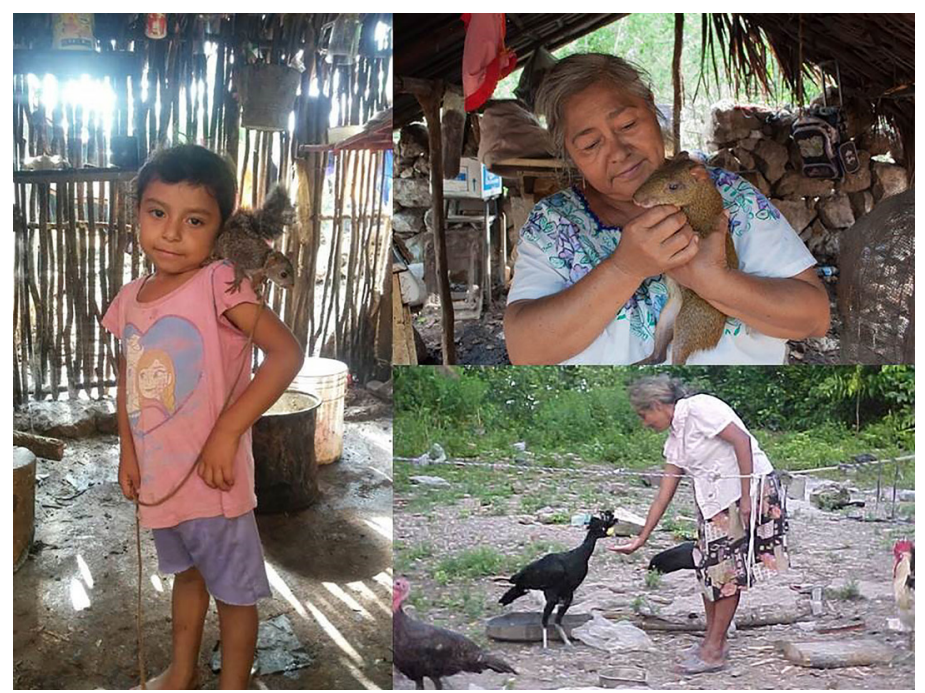

Figura 3. Animales del bosque criados como mascotas.

Wild forest animals adopted as pets. 


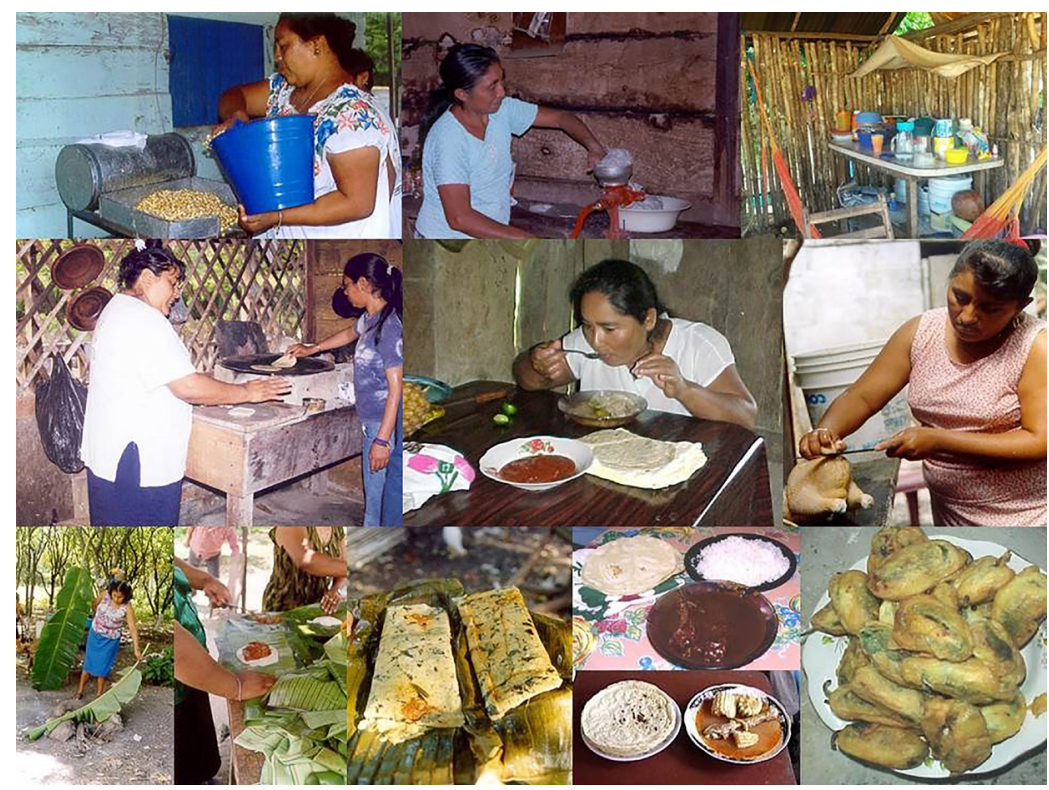

Figura 4. Cocinas, platillos y preparación de alimentos en los solares de Calakmul, Campeche.

Kitchens, platters, and backyard food preparation in Calakmul, Campeche.

recomendados por laFAO (2004). Tampoco se incrementó el promedio de Kcal compradas durante la temporada de escasez. Estos resultados fueron reveladores, pues sugieren que las consecuencias estacionales en el fenotipo no resultan de una "hambruna estacional", y que las tiendas de abarrotes no amortiguan sus efectos como sugieren Popkin y Gordon-Larsen (2004).

Aunque todos los grupos alimenticios de la clasificación de la FAO (Kennedy et al. 2013) están representados a lo largo del año, su abundancia es distinta en las dos temporadas y diferencias en biodiversidad pueden tener un impacto en la complementariedad de la dieta (Bioversity International 2011; Burchi et al. 2011; Heywood 2013). La mayoría de los alimentos estacionales solo están disponibles durante la temporada de abundancia. De hecho, la diferencia es ligeramente mayor de lo que presenta la Tabla 2. Algunos de los vegetales de la temporada de escasez se cosechan en su último mes, octubre, y por tanto están disponibles para su consumo en la temporada de abundancia que empieza a finales de octubre y principios de noviembre para darle la bienvenida a los finados y preparar los pibipollos ${ }^{3}$.

En Kenya, M'Kaibi et al. (2015) mostraron que la cantidad de hierro, calcio, zinc y ácido fólico consumidos por comunidades agrícolas se incrementaba significativamente durante su temporada de abundancia. Aunque en este estudio no se cuantificaron los micronutrientes, la presencia de una mayor cantidad de especies durante la temporada de abundancia en la Tabla 2, sugiere que, como en Kenya, en Calakmul también podría existir una merma estacional en la complementariedad de la dieta. Esta podría ser responsable de las pérdidas de peso, grasa y masa magra observadas durante la temporada de escasez por el autor principal en esta muestra (Alayón-Gamboa y Gurri 2007). La reducción en el consumo de alimentos bajos en calorías pero ricos en micronutrientes fue también observada por los autores entre campesinos de temporal en el estado de Yucatán (Gurri et al. 2018).

El uso de la flora y fauna local e introducida en la dieta de los campesinos de Calakmul es un ejemplo de adaptabilidad de una población que depende de su ambiente, para sobrevivir. Con gusto vemos que la introducción de las tienditas no ha afectado la generación en 20 años de una cocina local basada en los ingredientes que les ofrece el entorno. Estos incluyen elementos domesticados en la antigua Mesoamérica, plantas, y animales salvajes de la selva de Calakmul, y plantas y animales domésticos introducidos después de la conquista y que seguramente fueron importados a la región por los migrantes. También es un ejemplo más de la importancia de contribuir a preservar los espacios que sostienen la capacidad de los campesinos de hacer un manejo agroecológico exitoso y sustentable en un mundo globalizado (DeClerck et al. 2011). 
Cada localidad de Calakmul tiene acceso a una "tiendita" de abarrotes. Su existencia, sin embargo, no necesariamente presenta un obstáculo al uso del agroecosistema. Sus productos complementan en lugar de sustituir el sistema culinario local basado en los alimentos obtenidos del ambiente. Unos, literalmente le dan sabor al caldo como la sal, la pimienta, el comino y el aceite. Otros productos comprados se cosechan localmente reduciendo la necesidad de sustituirlos por alimentos globalizados. Entre estos productos se encuentra la harina de maíz (Maseca, Minsa) que no ha sustituido a la tortilla nixtamalizada, pero sí se usa cuando esta falta. Finalmente, tenemos otros cereales que se guisan con ingredientes locales como el arroz, aumentando con ello la diversidad de la dieta.

Las familias de Calakmul compran bebidas gaseosas, jarabes saborizantes, galletas de animalitos, frituras de maíz y golosinas. Esta oferta ha generado el gusto por alimentos industriales, pero dudamos que presenten un serio riesgo a la diversidad de la dieta local. Mientras su capacidad de producir y recolectar alimentos no sea socavada, y ni su sistema agroecológico sustituido por otros sistemas productivos, es muy probable que se desarrolle una tradición culinaria basada en los productos de su agroecosistema.

Agradecimientos: Los autores agradecemos a las 16 familias que nos dejaron entrar a sus casas y alterar su cotidianidad durante 24 horas, no una, sino tres veces durante el ciclo agrícola 2001-2. Un trabajo de esta naturaleza requirió de la colaboración de muchas personas durante 12 meses. Agradecemos a los becarios del proyecto, a los estudiantes del verano de la ciencia de la AMC y del programa Delfín que participaron con entusiasmo durante su estancia en Calakmul. Agradecemos a Karely Aragón y Paola Ruiz Becerra por su trabajo de depuración y sistematización de las bases de datos. Los datos recabados para este análisis se obtuvieron con financiamiento del proyecto 200000702017 de SISIERRA y del convenio CGCRB/CONABIOPromac/015/2018 con CONABIO. Finalmente, les damos las gracias a los evaluadores anónimos, cuyas sugerencias nos permitieron mejorar el manuscrito. Las opiniones y conclusiones aquí presentadas son responsabilidad exclusiva de los autores.

\section{Referencias Citadas}

Alayón-Gamboa, J.A. y F.D. Gurri 2007. Impact of seasonal scarcity on energy balance and body composition in peasant adolescents from Calakmul, Campeche Mexico. American Journal of Human Biology 19 (1):751-762.

Alayón-Gamboa, J.A. y F.D. Gurri-García 2008. Home garden production and energetic sustainability in Calakmul, Campeche, Mexico. Human Ecology 36 (3):395-407.

Baer, R.D. 1987. Nutritional effects of commercial agriculture. Urban Anthropology and Studies of Cultural Systems and World Economic Development 16 (1):39-61.

Baker, P. y S. Friel 2016. Food systems transformations, ultraprocessed food markets and the nutrition transition in Asia. Globalization and Health 12 (1):1-15.

Barthes, R. 2006. Por una psico-sociología de la alimentación contemporánea. EMPIRIA. Revista de Metodología de Ciencias Sociales 11:205-221.

Biing-Hwan, L., M. Ver Ploeg, P. Kasteridis y S.T. Yen 2014. The roles of food prices and food access in determining food purchases of low-income households. Journal of Policy Modeling 36 (5):938-952.

Bioversity International 2011. Improving Nutrition With Agricultural Biodiversity. Biodiversity International, Rome.

Burchi, F., J. Fanzo y E. Frison 2011. The role of food and nutrition system approaches in tackling hidden hunger. International Journal of Environmental Research and Public Health 8 (2):358-373.

Bustillos, B., J.R. Sharkey, J. Anding y A. McIntosh 2009. Availability of more healthful food alternatives in traditional, convenience, and nontraditional types of food stores in two rural Texas counties. Journal of the American Dietetic Association 109 (5):883-889.

Campeche.com.mx 2014. ¡Sin prisas! Aquí la receta para preparar el tradicional pibipollo. (25 mayo). https://www.campeche.com $\mathrm{mx} /$ sin-prisas-aqui-la-receta-para-preparar-el-tradicional-pibipollo/

Cantor, A.R., I. Chan y K. Baines 2018. From the chacra to the tienda: dietary delocalization in the peruvian andes. Food and Foodways 26 (3):198-222.

Chablé-Can, E., F.D. Gurri, D.O.R. Molina y B. Schmook 2007. Fuentes de ingreso y empoderamiento de las mujeres campesinas en el municipio de Calakmul, Campeche. Política y Cultura 28:71-95.

Chappell, M.J., H. Wittman, C.M. Bacon, B.G. Ferguson, L. García Barrios, R. García Barrios, D. Jaffee, J. Lima, V.E. Méndez, H. Morales, L. Soto-Pinto, J. Vandermeer e I. Perfecto 2013. Food sovereignty: an alternative paradigm for poverty reduction and biodiversity conservation in Latin America. F1000 Research 2 (235):1-18.

DeClerck, F.A.J., J. Fanzo, Ch. Palm, R. Remans 2011. Ecological approaches to human nutrition. Food and Nutrition Bulletin 32 (Suppl. 1):S41-S50.

DeWalt, K.M. 1993. Nutrition and the commercialization of agriculture: Ten years later. Social Science and Medicine 36 (11):1407-1416

Dewey, K.G. 1981. Nutritional consequences of the transformation from subsistence to commercial agriculture in Tabasco, Mexico. Human Ecology 9 (2):151-187. 
Entrena-Durán, F. y J.F. Jiménez-Díaz 2013. La producción social de los hábitos alimenticios. Una aproximación desde la sociología del consumo. Revista de Ciencias Sociales 19 (4):683-693.

Fanzo, J., D. Hunter, T. Borelli y F. Mattei (eds.) 2013. Diversifying Food and Diets: Using Agricultural Biodiversity to Improve Nutrition and Health. Routledge, London y New York.

Ferro-Luzzi, A., C. Scaccini, S. Taffese, B. Aberra y T. Demeke 1990. Seasonal energy deficiency in Ethiopian rural women. European Journal of Clinical Nutrition 44 (Suppl. 1):7-18.

Fleuret, P. y A. Fleuret 1980. Nutrition, consumption, and agricultural change. Human Organization 39 (3):250-260.

Golden, C.D., B. Vaitla, L. Ravaoliny, M.A. Vonona, E.J.G. Anjaranirina, H.J. Randriamady, R.P. Glahn, S.E. Guth, L.C.H. Fernald y S.S. Myers 2019. Seasonal trends of nutrient intake in rainforest communities of north-eastern Madagascar. Public Health Nutrition 22 (12):1-10.

Götz, C.M. 2014. La alimentación de los mayas prehispánicos vista desde la zooarqueología. Anales de Antropología 48 (1):167-199.

Gurri, F.D. 2007. Agriculture in Calakmul resiliency, sustainability or a better standard of living? Voices of Mexico 80:84-88.

Gurri, F.D. 2010. Smallholder land use in the southern Yucatan: how culture and history matter. Regional Environmental Change 10 (3):219-231.

Gurri, F.D. 2011. La doble carga de la transición nutrimental en zonas rurales de la península de Yucatán, ¿consecuencia de la alteración de los sistemas agrícolas de subsistencia tradicionales en la segunda mitad del siglo XX? En Obesidad Problema Multifactorial, editado por J.M. Muñoz Cano, pp. 65-84. Universidad Juárez Autónoma de Tabasco, Villahermosa.

Gurri, F.D., J.A. Alayón-Gamboa y D.O. Molina-Rosales 2002. Adaptabilidad en Poblaciones Mayas y Poblaciones Migrantes de Calakmul, Campeche. ECOSUR, Campeche.

Gurri, F.D., J.A. Alayón-Gamboa, D.O. Molina Rosales y M.I. Vallejo-Nieto 2004. Tablas de Valor Nutritivo de los Alimentos de Mayor Consumo en Calakmul, Campeche, México. ECOSUR, Campeche.

Gurri, F.D., W. Ruiz-García, E. Mena, D.O. Molina-Rosales y M.I. Vallejo-Nieto 2018. Impact of community development and abandonment of traditional subsistence strategies on the diet of maya populations from the maize region of Yucatan, Mexico. Congreso Internacional de Americanistas. Salamanca, España.

Guthman, J. 2011. Weighing in. Obesity, Food Justice and the Limits of Capitalism. University of California Press, Berkeley/Los Angeles,

Heywood, V.H. 2013. Overview of agricultural biodiversity and its contribution to nutrition and health. En Diversifying Food and Diets: Using Agricultural Biodiversity to Improve Nutrition and Health, editado por H. Fanzo J., T. Borell y F. Mattei, pp. 35-67. Routledge, London and New York.

Hirvonen, K., A.S. Taffesse y W.I. Hassen 2015. Seasonality and household diets in Ethiopia. Public Health Nutrition 13:1-8.

Instituto Nacional de Geografía e Informática [INEGI] 2010. Principales Resultados Por Localidad (ITER) del Censo de Población y Vivienda 2010, Campeche (25 mayo). https://datos.gob.mx/busca/dataset/censo-de-poblacion-y- vivienda-2010-principales-resultados-por-localidad-iter/ resource/9d898de9-e301-4837-be78-96fdf6633b4a?inner_ span $=$ True

Instituto Nacional de Ciencias Médicas y Nutrición Salvador Zubirán (INCMNSZ) 1998. Tabla de Composición de Alimentos Mexicanos. INCMNSZ, México DF.

Jacques, P.J. y J.R. Jacques 2012. Monocropping cultures into ruin: The loss of food varieties and cultural diversity. Sustainability 4 (12):2970-2997.

Kennedy, E., H. Bouis y J. von Braun 1992. Health and nutrition effects of cash crop production in developing countries: A comparative analysis. Social Science \& Medicine 35 (5):689-697.

Kennedy, G., T. Ballard y M.C. Dop 2013. Guía para Medir la Diversidad Alimentaria a Nivel Individual y del Hogar. FAO, Roma.

Kraft, T.S., J. Stieglitz, B.C. Trumble, M. Martin, H. Kaplan y M. Gurven 2018. Nutrition transition in 2 lowland Bolivian subsistence populations. American Journal of Clinical Nutrition 108 (6):1183-1195.

Leatherman, T.L. y A. Goodman 2005. Coca-colonization of diets in the Yucatan. Social Science and Medicine 61 (4):833-846.

Leonard, W.R. 1991. Household-level strategies for protecting children from seasonal food scarcity. Social Science and Medicine 33 (10):1127-1133.

Lieberman, L. 1986. Nutritional anthropology at the household level. En Training Manual in Nutritional Anthropology, editado por S. Quandt y C. Ritenbaugh, pp. 21-40. American Anthropological Association, Washington D.C.

Liese, A.D., K.E. Weis, D. Pluto, E. Smith y A. Lawson 2007. Food store types, availability, and cost of foods in a rural environment. Journal of the American Dietetic Association 107 (11):1916-1923.

Lope-Alzina, D.G. 2017. Cuatro décadas de estudio en huertos familiares Maya-Yucatecos: hacia la comprensión de su variación y complejidad. Gaia Scientia 11 (3):160-184.

M'Kaibi, F.K., N.P. Steyn, S. Ochola, L. Du Plessis, E. Cromwell, D. Cooper y T. Hodgkin 2015. Effects of agricultural biodiversity and seasonal rain on dietary adequacy and household food security in rural areas of Kenya. BMC Public Health 15 (1):422.

Mariaca-Méndez, R. 2015. La milpa maya yucateca en el siglo XVI: evidencias etnohistóricas y conjeturas. Etnobiología 13 (1):1-25.

Marín Cárdenas, A., G. Sánchez-Ramírez y L. Maza 2014. Prevalencia de obesidad y hábitos alimentarios desde el enfoque de género: el caso de Dzutóh, Yucatán, México. Estudios Sociales. Revista de investigación científica 22 (44):65-90.

Martínez, E. y C. Galindo Leal 2002. La vegetación de Calakmul, Campeche, México: Clasificación, descripción y distribución. Boletín de la Sociedad Botánica de México 71 (4):7-32.

Mc Phail Fanger, E. 2012. Publicidad, hábitos y motivaciones. Razón y palabra 17 (80):1-25.

Messer, E. 1977. The ecology of vegetarian in a modernizing mexican community. En Nutrition and Anthropology in Action, editado por T. Fitzgerald, pp. 117-124. Van Gorcum, Amsterdam.

Monteiro, C.A., R.B. Levy, R.M. Claro, I.R.R. De Castro y G. Cannon 2011. Increasing consumption of ultra-processed foods 
and likely impact on human health: Evidence from Brazil. Public Health Nutrition 14 (1):5-13.

Neulinger, K., C.R.,Vog1 y J.A. Alayón-Gamboa 2013. Plant species and their uses in homegardens of migrant maya and mestizo smallholder farmers in Calakmul, Campeche, Mexico. Journal of Ethnobiology 33 (1):105-124.

Oomen, H. 1971. Ecology of human nutrition in New Guinea: Evaluation of subsistence patterns. Ecology of Food and Nutrition 1 (1):1-6.

Organización de las Naciones Unidas para la Agricultura y la Alimentación [FAO] 2004. Human Energy Requirements. Report of a Joint FAO/WHO/UNU Expert Consultation, Roma 2001. FAO - World Health Organization - United Nations University, Roma.

Otero, D.E., F.D. Gurri, R. Mariaca y F. Guízar 2017. La incorporación y el aumento de oferta de alimentos industrializados en las dietas de las unidades domésticas y su relación con el abandono del sistema de subsistencia propio en las comunidades rurales mayas de Yucatán, México. Cuadernos de Desarrollo Rural 14 (80):1-16.

Perea Mercado, S.L. 2011. Influencia del Empoderamiento de las Mujeres sobre la Diversidad Vegetal en Solares de Campeche, México. Tesis de Licenciatura en Antropología Social. Facultad de Antropología, Universidad Autónoma del Estado de México, México DF.

Pérez Izquierdo, O., A. Nazar Beutelspacher, B. Salvatierra Izaba, S.E. Pérez-Gil Romo, L. Rodríguez***, M.T. Castillo Burguete, R. Mariaca Méndez 2012. Frecuencia del consumo de alimentos industrializados modernos en la dieta habitual de comunidades mayas de Yucatán, México. Estudios Sociales 20 (39):155-184.

Popkin, B.M. y P. Gordon-Larsen 2004. The nutrition transition: worldwide obesity dynamics and their determinants. International
Journal of Obesity and Related Metabolic Disorders 28 (Suppl 3):S2-9.

Secretaría de Desarrollo Social [SEDESOL] 2013. Cédula de Información Municipal (SCIM) Campeche, Calakmul. (4 septiembre). http://www.microrregiones.gob.mx/zap/datGenerales. asp $x$ ?entra $=$ pdzp\&ent $=04 \&$ mun $=010$

Secretaria de Salud [SSA] 2013. Norma Oficial Mexicana NOM-043-SSA2-2012, Servicios básicos de salud. Promoción y educación para la salud en materia alimentaria. Criterios para brindar orientación. Diario Oficial de la Federación. Martes 22 de enero de 2013:1-29.

Sharkey, J.R., W.R. Dean y C. Nalty 2012. Convenience stores and the marketing of foods and beverages through product assortment. American Journal of Preventive Medicine 43 (Suppl.1):S109-115.

Sharkey, J.R., D.W. Dean, C. Nalty y J. Xu 2013. Convenience stores are the key food environment influence on nutrients available from household food supplies in Texas Border Colonias. Public Health 13 (45):1-8.

Sohn, Y.S., E. Moran y F. Gurri1999. Deforestation in northcentral Yucatan (1985-1995): Mapping secondary succession of forest and agricultural land use in Sotuta using the cosine of the angle concept. Photogrammetric Engineering and Remote Sensing 65 (8):947-958.

Terán, S. y C.H. Rasmussen 2009. La Milpa de los Mayas: La Agricultura de los Mayas Prehispánicos y Actuales en el Noroeste de Yucatán. 2da.ed., UNAM, Universidad de Oriente, Mérida.

Varela Scherrer, C.M. y F. Trabanino 2017. La cacería tradicional chol y tseltal en los acahuales de Palenque: implicaciones para la zooarqueología maya. Pueblos y Fronteras 11 (22):165-191.

\section{Notas}

1 Tierra comunal, otorgada a campesinos después de la reforma agraria. El campesino la puede trabajar y usar su producto, pero no la puede vender.

2 Policultivo tradicional de Mesoamérica basado en el maíz. Aunque varía por región, suele combinar frijol, calabaza y chile.

3 Es el nombre en idioma maya yucateco de un guiso preparado a partir de masa de maíz nixtamalizado, grasa de cerdo, pollo

y diversos condimentos (recado de achiote, pimientas de Castilla y Tabasco, ajo, epazote, jitomate y chile habanero), con lo cuales se forma una especie de tamal grande, envuelto en hojas de plátano y cocido lentamente bajo la tierra a la usanza maya. Este guiso es tradicional del Día de Muertos. Se acostumbra acompañar con atole nuevo y chocolate. Se prepara en Campeche y Yucatán. Conocido también como mukbil pollo (Campeche.com.mx 2014). 
